ISSN: 2174-5609

DOI. http://dx.doi.org/10.14198/INTURI2017.13.02

TJT Investigaciones

\title{
La experiencia de la calidad de servicio online como antecedente de la satisfacción online: estudio empírico en los sitios web de viajes
}

\author{
José Ramón Sarmiento Guede \\ joseramonsarmiento@gmail.com \\ Universidad Internacional de La Rioja y ESERP Business School. España
}

\section{RESUMEN}

El presente trabajo de investigación tiene por objeto conocer la relación entre las principales dimensiones que forman la calidad de servicio online (experiencia de compra, diseño, seguridad, información y comunicación) y la satisfacción de los usuarios de los sitios web de viajes. La metodología utilizada consiste en entrevistas en profundad y en la revisión de la bibliografía para identificar las dimensiones aislables como conformadoras de la calidad de servicio y de la satisfacción. Una vez propuesto el modelo conceptual, utilizamos la técnica de la encuesta para poder medir cada uno de los ítems. Para los resultados, se utiliza el análisis factorial para la fase exploratoria y las ecuaciones estructurales para la confirmatoria. Como conclusión, podemos afirmar que existe una relación positiva entre las dimensiones que forman la calidad de servicio en los sitios web de viajes y la satisfacción de los usuarios, en concreto, las dimensiones de la comunicación y de la información tienen un papel determinante en la satisfacción de los servicios de los sitios web de viajes.

Palabras clave: calidad de servicio; satisfacción; experiencia online; medios sociales; marketing de relaciones 


\title{
The experience of online service quality as a precedent for online satisfaction: An empirical study on travel websites
}

\begin{abstract}
This research project aims to identify the relationship between the principal dimensions that contribute to ensuring the quality of online service (shopping, design, security, information and communication experience) and the satisfaction of the users of travel websites. The methodology used consists of in-depth interviews and a review of the literature to identify the dimensions that can be identified as comprising the quality of service and satisfaction. After proposing the conceptual model, the survey technique is used to measure each of the items. For the results, the factorial analysis is used for the exploratory phase and the structural equations for the confirmatory phase. In conclusion, we can affirm that there is a positive relationship between the dimensions that comprise the quality of service in travel websites and the satisfaction of users. Specifically, the dimensions of communication and information have a determining role in the satisfaction of the services of travel websites.
\end{abstract}

Keywords: service quality; satisfaction; online experience; social media; relationship marketing

\section{INTRODUCCIÓN}

En los últimos años, la experiencia online ha sido un tema crucial para el marketing, y podemos decir que también para el sector turístico. Y la experiencia del sitio web parece que no es definible solamente como la percepción total del consumo de una empresa en línea, sino como el resultado de su exposición a una combinación de las herramientas de marketing. Esta idea está reafirmada por Cristóbal et al. (2007) y por Chung y Shin (2010), quienes ya señalaron la necesidad de una revisión del servicio para adaptarlo al mundo virtual. $\mathrm{Y}$, con este fin, realizaron diversas investigaciones en su mayoría para desarrollar escalas de medición adaptadas al medio (Internet).

Hasta ahora la mayoría de los usuarios utilizaba servicios offline para planificar los viajes, pero en los últimos años las estadísticas ofrecidas por los organismos oficiales señalan que cada vez recurren más a los servicios online para ello. $\mathrm{Y}$ el principal gap que encontramos es que la mayor parte de los trabajos sobre la calidad de servicio y satisfacción centró su interés en un contexto offline. Por ello, ante la escasa bibliografía sobre este aspecto y ante el constante incremento de uso de los sitios web de viajes online, se echa en falta una revisión y adaptación de las dimensiones que conforman la calidad del servicio online y la satisfacción online, aspectos que por sí solos justifican el propósito de nuestro estudio.

En esta investigación, asumimos que la calidad de servicio online ejerce un efecto positivo sobre la satisfacción online (Athanasopoulou, 2009), pero desconocemos todavía qué dimensiones de la calidad del servicio del sitio web son las que influyen más significativamente sobre la satisfacción en el contexto digital. 
En consecuencia, esta investigación se propone como objetivo principal analizar la influencia de la calidad de servicio online sobre la satisfacción online, incluyendo los factores que forman la calidad de servicio tales como la comunicación, el diseño, la seguridad, la información, la usabilidad y la interactividad.

A este objetivo genérico, subyace una serie de objetivos específicos que enumeramos a continuación:

- Analizar e identificar las dimensiones que conforman la calidad de servicio.

- Analizar las definiciones publicadas sobre la calidad de servicio online y determinar cuál de ellas es la más acertada.

- Identificar los sitios web de viajes y establecer una clasificación.

Para lograr estos objetivos, estructuramos el trabajo de la manera siguiente: en primer lugar, ofreceremos una revisión de la literatura disponible sobre la calidad del servicio online y la satisfacción online; en segundo lugar, y, tomando como base dicha revisión, analizaremos y constataremos los resultados obtenidos mediante técnicas cualitativas y cuantitativas. $Y$, por último, presentaremos las conclusiones en las que discutiremos si hemos logrado los principales objetivos y si hemos podido probar las hipótesis.

\section{MARCO TEÓRICO Y PLANTEAMIENTO DE LAS HIPÓTESIS}

Así pues, en lo que sigue daremos cuenta de las dimensiones de estudio de la calidad del servicio y la satisfacción del cliente y también formularemos las hipótesis para constatarlas posteriormente.

\subsection{Calidad de servicio online}

Para elaborar una definición clara de la calidad de servicio, Parasuraman et al. (1985, p. 42) asumieron las premisas siguientes:

- Que la calidad de servicio es más difícil de evaluar para los consumidores que la calidad de un producto o un bien.

- Que las percepciones de la calidad de servicio nacen de la comparación de las expectativas del consumidor respecto del funcionamiento real de servicio.

- Que las evaluaciones de calidad no se realizan únicamente sobre el resultado del servicio, porque también implican evaluaciones sobre el proceso de prestación de servicios.

A partir de estas premisas, Parasuraman et al. (1985, p. 16) concluyeron que la percepción de la calidad de servicio "es el juicio global o la actitud en relación con la superioridad del servicio". Este enfoque de la calidad, por ser más subjetivo y estar basado en las percepciones del cliente, alcanza un mayor protagonismo en las investigaciones realizadas en el Marketing de Servicios (Grönroos, 1984). Así, Zeithmal (1988) definió "la calidad de servicio como la valoración que el cliente hace de la excelencia o superioridad del servicio" (Setó, 2004, p. 17). En cambio, Parasuraman, Zeithaml y Berry $(1985,1988)$ afirmaron que la calidad de servicio viene determinada por la diferencia entre las expectativas del cliente sobre el rendimiento de los proveedores de servicios y la evaluación de los servicios recibidos. Lo cual nos lleva a pensar que la definición de calidad de servicio 
debería incluir, según Grönroos (1983) y Parasuraman et al. (1988), la comparación entre lo que el cliente espera recibir y lo que realmente recibe o percibe que ha recibido. Se trata de un concepto altamente subjetivo, similar al de actitud (Bitner, 1990; Zeithaml, 1988), pues implica un proceso de evaluación continuada en el tiempo. Según Oliver (1981, p. 41), la actitud es "una orientación afectiva hacia un objeto y no implica sorpresa como concepto central". Por eso, Bolton y Drew (1991, p. 2) afirman que "la actitud del cliente corresponde a una evaluación global del producto/servicio más que a una evaluación de una transacción específica". Esto explica que haya muchos autores que identifiquen la calidad de servicio con la actitud y que ambas cosas las definan de igual modo (Bitner, 1990) o que haya otros autores que conceptualicen la calidad de servicio como una clase de actitud, cuando son claramente conceptos distintos. En consecuencia, en nuestra investigación asumimos que la definición de la calidad de servicio implica una comparación entre lo que el cliente espera recibir como expectativas antes del servicio y lo que realmente ha recibido como desempeño del resultado del servicio.

En suma, la principal diferencia entre los servicios offline ofrecidos en un contexto analógico y la prestación de servicios online ofrecidos en un contexto virtual es la sustitución de las interacciones persona-persona por las interacciones usuario-ordenador (Evanschitzky et al., 2004). Mientras en los servicios offline hay personas únicamente implicadas en los encuentros de servicio, en los servicios online se da una interacción entre las TIC y los usuarios gracias a la mediación de Internet (Surjadjaja et al., 2003).

Boyd et al. (2002, p. 175), por servicios online, entendieron "todos los servicios interactivos que se desarrollan en Internet mediante las telecomunicaciones, información y tecnologías multimedia". Un año más tarde, Santos (2003, p. 235) definió la calidad online como "las evaluaciones y consideraciones que los consumidores realizan sobre la excelencia y calidad de la oferta de los servicios electrónicos en el marketspace". Partiendo de estas dos concepciones sobre los servicios online y la calidad online, Parasuraman et al. (2005, p. 217) llegaron a interpretar la calidad de servicio online como "la medida en que un sitio web facilita de forma eficiente y eficaz el proceso de compra, la propia compra y la entrega de productos o servicios".

Sin embargo, por Fassnacht y Koese (2006) criticaron esta definición; adujeron que abarcaba solo un campo muy estrecho de los servicios online; en concreto, todo lo referente a las compras por Internet. Así, desde una perspectiva más amplia, Cristóbal et al. (2007) señalaron que los diversos estudios sobre la calidad de servicio online podían agruparse en dos grandes categorías:

- Servicios de venta en línea detallista.

- El diseño y la calidad del sitio web.

Entre los estudios de la primera categoría, cabe mencionar el modelo e-SERVQUAL desarrollado por Zeithaml et al. (2013). En él, identificaron 11 dimensiones sobre la calidad de servicio online; fueron las siguientes (Stiakakis y Georgiadis, 2009): (1) acceso (al sitio web cuando sea necesario); (2) aseguramiento / confianza (que el cliente confíe en el sitio); (3) facilidad de navegación (que se mueva fácilmente y con rapidez a través de las páginas del sitio web); (4) eficiencia (que el sitio sea fácil de usar), (5) flexibilidad (en la realización de una transacción electrónica); (6) personalización / personalizar (con base en las preferencias 
del cliente y el historial de compra); (7) conocimiento del precio (relativo al total, al envío y los precios comparativos); (8) seguridad / privacidad (si el sitio web está a salvo de intrusos y la información personal, protegida); (9) la estética del sitio (atributos de apariencia); (10) confiabilidad (correcto funcionamiento técnico del sitio, cumplir las promesas hechas a los clientes); (11) capacidad de respuesta (rápida a las necesidades del cliente).

En la segunda categoría sugerida por Cristóbal et al. (2007), resulta evidente que el diseño del sitio web es la dimensión más frecuentemente examinada por los investigadores (Barnes y Vidgen, 2002). En este sentido, Loiacono (2000) desarrolló una escala denominada WebQual formada por 12 dimensiones (Stiakakis y Georgiadis, 2009): (1) información adecuada para la tarea; (2) interacción; (3) confianza; (4) tiempo de respuesta; (5) facilidad de comprensión; (6) operaciones intuitivas; (7) atractivo visual; (8) carácter innovador; (9) atractivo emocional; (10) imagen coherente; (11) integridad en línea; (12) ventaja comparativa.

Otro de los modelos digno de mención y más reciente es el desarrollado por Cristóbal et al. (2007); en él, estos autores pudieron medir mediante la escala PeSQ la calidad de servicio percibida por los clientes de establecimientos en línea. Y entre los factores más importantes para desarrollar el diseño de los sitios web señalan los siguientes:

- La facilidad de la comprensión de la estructura de un sistema, sus funciones, la interfaz y los contenidos que puedan ser observados por el usuario.

- La sencillez de uso del sitio web en sus etapas iniciales.

- La velocidad con que los usuarios pueden encontrar lo que están buscando.

- La facilidad de navegación del sitio web percibida en términos de tiempo.

- La capacidad del usuario para controlar lo que está haciendo y dónde se encuentra en todo momento.

Con el desarrollo del marketspace, la unidad de negocio físico ha sido sustituida por un sitio web (Cristóbal et al., 2007). De los estudios analizados, parece deducirse que en el marketspace la percepción de la calidad de servicio online constituye una parte muy importante de la imagen de la tienda, porque puede influir tanto en la actitud como en el comportamiento de compra de los usuarios en línea. De hecho, la percepción del diseño del sitio web juega un papel relevante a la hora de ofrecer servicios a los consumidores en línea. En consecuencia, Kim y Eom (2002) llegaron a la conclusión de que los diseños de sitios web son de importancia capital para el logro de la satisfacción global del usuario. No obstante, consideramos que la literatura existente sobre la calidad de servicio online es mejorable mediante estudios sobre el servicio de sitios web, el diseño y la usabilidad. Es necesario constatar también su relación e influencia sobre la satisfacción, la confianza, el compromiso y los comportamientos del usuario.

\subsection{Satisfacción online}

"Todos saben qué es la satisfacción hasta que se les pide una definición. Entonces, al parecer, nadie lo sabe" (Zeithmal et al., 2009, p.104). Traemos a colación esta cita de un experto investigador sobre la satisfacción, porque expresa bien el desafío que supone definir dicho concepto. En efecto, basándose en definiciones previas, Oliver elaboró otra sobre lo que se entiende por satisfacción en estos términos: es "la respuesta de realización del consumidor. Es un juicio sobre una característica del producto o servicio, o el producto o 
servicio en sí, que proporciona un nivel placentero de realización relativa al consumo" (Oliver, 1997, p. 17).

Tabla 1. Resumen de las características que determinan la satisfacción online

\begin{tabular}{|c|c|}
\hline Autores & Determinantes de la satisfacción online \\
\hline $\begin{array}{l}\text { Szymanski y Hise } \\
(2000)\end{array}$ & $\begin{array}{l}\text { 1) Conveniencia; 2) Facilidad de uso/Usabilidad; 3) Diseño del sitio web, 4) Seguridad; 5) } \\
\text { Atención al cliente; 6) Variedad de productos/servicios; 7) Información. }\end{array}$ \\
\hline $\begin{array}{l}\text { Kim y Lim } \\
(2001)\end{array}$ & 1) Conveniencia; 2) Confiabilidad; 3) Velocidad; 4) Información; 5) Entretenimiento. \\
\hline $\begin{array}{l}\text { Devaraj et al. } \\
\text { (2002) }\end{array}$ & 1) Facilidad de uso/Usabilidad; 2) Garantía; 3) Utilidad. \\
\hline $\begin{array}{l}\text { Yoon } \\
(2002)\end{array}$ & 1) Seguridad; 2) Propiedades del sitio web; 3) Navegación; 4) Personalización. \\
\hline $\begin{array}{l}\text { Heiner et al. } \\
(2004)\end{array}$ & $\begin{array}{l}\text { 1) Conveniencia; 2) Diseño del sitio web; 3) Seguridad; 4) Variedad de } \\
\text { productos/servicios; 5) Información. }\end{array}$ \\
\hline $\begin{array}{l}\text { Kim y Stoel } \\
(2004)\end{array}$ & $\begin{array}{l}\text { 1) Diseño del sitio web; 2) Confianza; 3) Información; 4) Respuesta a tiempo; 5) } \\
\text { Capacidad de transacción; 6) Entretenimiento. }\end{array}$ \\
\hline $\begin{array}{l}\text { Bansal et al. } \\
(2004)\end{array}$ & $\begin{array}{l}\text { 1) Facilidad de uso/Usabilidad; 2) Precio; 3) Duración de la transacción; 4) Servicio al } \\
\text { cliente; 5) Gastos de envío; 6) Información. }\end{array}$ \\
\hline $\begin{array}{l}\text { Hsuehen } \\
(2006)\end{array}$ & 1) Diseño del sitio web; 2) Información. \\
\hline $\begin{array}{l}\text { Cristóbal et al. } \\
\text { (2007) }\end{array}$ & 1) Diseño del sitio web; 2) Garantía; 3) Servicio al cliente; 4) Gestión de pedidos. \\
\hline $\begin{array}{l}\text { Casaló et al. } \\
(2008)\end{array}$ & 1) Facilidad de uso/Usabilidad. \\
\hline $\begin{array}{l}\text { Herington } \quad y \\
\text { Weaven (2008) }\end{array}$ & $\begin{array}{l}\text { 1) Facilidad de uso/Usabilidad; 2) Eficiencia; 3) Necesidades personales; 4) Organización } \\
\text { del sitio web. }\end{array}$ \\
\hline $\begin{array}{l}\text { Kassin y Abdullah } \\
\text { (2010) }\end{array}$ & $\begin{array}{l}\text { 1) Facilidad de uso/Usabilidad; 2) Diseño del sitio web; 3) Garantía; 4) Personalización; } \\
\text { 5) Sensibilidad. }\end{array}$ \\
\hline $\begin{array}{l}\text { Sheng y Liu } \\
\text { (2010) }\end{array}$ & 1) Accesibilidad; 2) Eficiencia; 3) Cumplimiento; 4) Privacidad. \\
\hline
\end{tabular}

La investigación académica ha entendido normalmente la evaluación de la satisfacción offline del cliente como la confirmación de sus expectativas (Oliver 1997). En el modelo multi-atributo de la satisfacción del cliente (Oliver 1997; Mittal et al., 1998), un consumidor compara el rendimiento percibido de un atributo con una expectativa preconsumo formada a partir de la experiencia de compras anteriores y de la información externa. La satisfacción general es una función de amplitud de las múltiples no confirmaciones del atributo que intermedia con sus efectos sobre las intenciones de comportamiento. 
Hemos mencionado anteriormente que la diferencia más obvia entre los servicios offline y online consiste en la sustitución de la interacción humano-humano por la interacción usuario-computadora; por consiguiente, este cambio requiere nuevos enfoques para medir la satisfacción (Shankar et al., 2003), puesto que el consumidor en línea es a la vez comprador y usuario de la computadora (Koufaris 2002). Los consumidores online no pueden utilizar los cinco sentidos para tomar decisiones de compra en línea, sino que se enfrentan a representaciones limitadas como fotografías, vídeos y descripciones de texto. Por lo tanto, las decisiones en línea también son sensibles a los diseños de los sitios web y al desarrollo de las funciones de red como los sistemas de recomendación y el golpe de un solo clic (Koufaris 2002). Mientras que el tema de la satisfacción offline ha sido ampliamente discutido (Oliver, 1981; Anderson et al., 1994; Johan, 2006; Ofir y Simonson, 2007), la identificación de las características que determinan la satisfacción online está todavía en una fase incipiente (Heiner et al., 2004).

Durante los últimos años, algunos estudiosos han comenzado a investigar cómo pueden influir las características de un sitio web en la satisfacción electrónica de los clientes, pero, en este intento por construir un marco de evaluación o por identificar las características dominantes sobre la satisfacción electrónica desde la perspectiva de la calidad del sitio web (Ranganathan y Ganapathy, 2002), del comportamiento de compra (Koivumäki, 2001; Park y Kim, 2003), de la actitud de los consumidores (Elliott y Speck, 2005), del valor del cliente (Shun y Yunjie, 2006) y de la calidad del servicio (Zhilin et al., 2003), todavía no se ha llegado al consenso de sentar cómo afecta esto a la satisfacción online (Schaupp y Belanger, 2005) porque las aplicaciones tecnológicas varían y evolucionan de año en año.

La doble identidad del consumidor en línea como un comprador tradicional y un usuario de la computadora significa que atraer y retener a los clientes no depende solo de atractivo comercial en un contexto offline, sino también de la prestación de apoyo técnico constante en un contexto online (Straub y Watson, 2001). El cliente en línea no solamente forja expectativas sobre la mezcla de Marketing Mix (precios, productos, plaza y promoción), sino que también es depositario de expectativas sobre los problemas del sistema tales como la velocidad de carga. Pero, para comprender plenamente la satisfacción del cliente en línea, hay que entender la interacción de los usuarios con el sitio web como un almacén y como interfaz del sistema (Finn et al., 2009).

La literatura anterior viene a apoyar la idea de que la percepción de calidad de servicio lleva a mejorar la satisfacción del cliente, pues la calidad del servicio es un antecedente de la satisfacción. La justificación de esta conexión está en que la satisfacción del cliente es una reacción afectiva surgida como respuesta a una prolongada serie de encuentros cognitivos de servicio. Por lo tanto, autores como Sivadas y Prewitt (2000) señalan que la satisfacción del cliente es una experiencia pos-consumo que compara la calidad percibida con la calidad esperada. Szymanski y Hise (2000) entendieron la satisfacción online como un constructo global que refleja el efecto acumulado de un conjunto de experiencias discretas con el proveedor de servicios durante un período de tiempo. $\mathrm{Y}$, como tal, se mide el grado en el que un cliente está a la vez satisfecho e insatisfecho con las experiencias online. Cristóbal et al. (2007) afirmaron que durante los últimos años se ha demostrado que la calidad de servicio online tiene un efecto positivo en la satisfacción online y, posteriormente, en la rentabilidad de la empresa (Anderson et.al., 1994). 
También Anderson y Srinivasan definieron la satisfacción online como "la satisfacción del cliente respecto de su experiencia de la compra anterior con una determinada empresa del comercio entrópico" (2003, p. 125). Y, teniendo en cuenta que un cliente en línea no es simplemente un comprador, sino también un usuario de la tecnología de la información (Cho y Park, 2001), podemos afirmar que la experiencia online es la impresión total que un consumidor obtiene sobre una empresa online como resultado de su exposición a una combinación de herramientas virtuales de Marketing, experiencia que puede influir en el comportamiento del consumidor online (Constantinides, 2002, p.60). Por consiguiente, se puede concluir que la satisfacción online es "el juicio que realiza el cliente sobre experiencias habidas en relación con la percepción de una empresa en línea".

\subsection{Sitios web de viajes}

Internet ha modificado la forma de intercambiar información y de planificar y consumir viajes dentro del sector turístico (Buhalis y Law, 2008). Según Xiang y Gretzel (2009), son dos mega-tendencias las que han transformado el sistema turístico. Por un lado, están (a) los Medios Sociales (blogs, comunidades, wikis, redes sociales, mundos virtuales, etc.) que representan diferentes formas de generar contenido por el usuario (Gretzel, 2006; Pan et al., 2007). Pues los Medios Sociales facilitan a los consumidores el publicar y compartir sus comentarios sobre viajes, el difundir opiniones y experiencias personales que a su vez sirven de información para otros en tanto forma de retratar, reconstruir y revivir los viajes (Pudliner, 2007). Y, por otro lado, están (b) los sitios web turísticos en los que buscamos información para planificar un viaje, ya que su uso predomina entre los consumidores en Internet (Xiang y Gretzel, 2009). Así, los sitios web turísticos y los Medios Sociales han pasado a ser una importante interfaz a través de la cual las empresas turísticas pueden llegar a los visitantes potenciales y persuadirlos.

Por tanto, es fundamental tener presentes los cambios producidos con el uso de las nuevas tecnologías, pues permiten explicar cómo afectan al comportamiento y a la distribución de información sobre viajes (Fesenmaier, Wöber y Werthner, 2006; Xiang et al., 2008). No obstante, muchos investigadores coinciden en afirmar que falta todavía investigación empírica sobre el papel que desempeñan los sitios web de viajes. Es más, según diversos estudios (Walle, 1996; Kasavana et al., 1997), Internet pasa por ser una de las herramientas más eficaces de marketing, utilizada en sector turístico desde 1997 (Connolly et al., 1998) para hacer más accesible la información al usuario a través de los sitios web y personalizarla (Lin, 2010).

En consecuencia, Internet $y$, particularmente, los sitios web de viajes se han convertido en una de las plataformas más importantes para la industria de viajes en España; gracias a ellas, las empresas pueden prestar un servicio adicional e intercambiar información con sus clientes potenciales. Según Vázquez-Casielles et al. (2009), las agencias de viajes online son un sector caracterizado por una fuerte competitividad, que comercializa servicios perecederos y que, en muchos casos, tiene una alta estacionalidad en donde la calidad percibida por el cliente constituye el elemento clave de éxito.

$Y$, aunque algunas empresas surgen en el mundo digital y a éste limitan su actividad, lo más común es que una agencia de viajes offline cuente con otra en formato digital o que figure en páginas web para informar de su oferta, para reorientar y fortalecer su posición en los mercados actuales (Vázquez-Casielles et al., 2009). Una empresa que preste servicios 
online ha de estar siempre orientada hacia los clientes; debe ver las cosas a través de ellos para poder satisfacer mejor sus necesidades $y$, por tanto, necesita diseñar sistemas de servicios online que cumplan con sus expectativas. Así pues, identificar y comprender cómo los clientes definen de la calidad del servicio online es de importancia capital para una empresa que quiera desarrollar y saber gestionar relaciones en la aldea global de McLuhan. Internet constituye una plataforma de importancia vital no sólo para las agencias de viajes online, sino también para la industria de los proveedores (hoteles, transporte, atracciones, etc.), intermediarios (agencias de viajes), controladores (gobiernos, órganos administrativos, etc.), así como para otras muchas organizaciones sin fines de lucro.

Tabla 2. Clasificación de los sitios web de viajes

Nombre de los sitios web y referencia

Sitios web de alojamiento

Sitios web de transporte

Sitios web de agencias de viajes

Sitios web de motores de búsqueda

Sitios web de destinos

Sitios web de opinión
Ejemplos de sitios web de viajes

NH hoteles, AC hoteles, Sol Meliá
Iberia, Ryanair, Aireuropa, Renfe, Alsa
Viajes El Corte Inglés, Halcón Viajes, Barceló Viajes,
Booking, Rumbo, Atrápalo, Lastminute
Kayak, Trivago, Rastreator
Spain.info, Andalucia.org, Barcelona.turisme
Tripadvisor.com, Minube.es

NH hoteles, AC hoteles, Sol Meliá

Iberia, Ryanair, Aireuropa, Renfe, Alsa

Booking, Rumbo, Atrápalo, Lastminute

Tripadvisor.com, Minube.es

Elaboración propia a partir de Vallespín y Molinillo (2014) y Sarmiento (2016).

\subsection{La relación entre la calidad de servicio online y la satisfacción online}

En el entorno online, la satisfacción desempeña un papel muy importante. Son numerosos los modelos teóricos y prácticos que han considerado la calidad de servicio online como un factor determinante en la satisfacción online (Chung y Shin, 2010). En esta investigación, asumimos la calidad de servicio online como un antecedente de la satisfacción online (Athanasopoulou, 2009). Y, para probarlo, traemos a colación diferentes estudios que describimos a continuación.

Fue Oliver (1993) quien sugirió por primera vez que la calidad del servicio debe ser considerada como un antecedente de la satisfacción del cliente, independientemente de si estos constructos eran acumulativos o eran específicos. La satisfacción y la calidad del servicio presentan ciertas notas en común, pero la satisfacción, generalmente, es un concepto más amplio, mientras que la calidad del servicio se aplica más específicamente a las dimensiones del servicio (Wilson et al., 2008).

Mills y Morrison (2004) investigaron los atributos que potencian la satisfacción de los clientes en los sitios web de viajes y constataron que la calidad del servicio percibida se basa en un constructo multidimensional que desempeñaba un papel crucial en la determinación de la satisfacción del cliente. En este estudio, damos cuenta de algunas características del sitio web como el acceso, la velocidad de carga, la apariencia, la navegación, la interactividad, la búsqueda y la seguridad.

Ho y Lee (2007) también plantearon un modelo de investigación basado en la percepción de la calidad de servicio online y su influencia en la satisfacción online. Para ello, utilizaron características como la calidad de la información, seguridad, funcionalidad de sitio web, relaciones con los clientes, capacidad de respuesta. 
Cristóbal et al. (2007) consideraron que los aspectos para medir la calidad de servicio en Internet era un campo de estudio aún en una fase incipiente (Van Riel et al., 2001). Por consiguiente, con su escala intentaron medir aspectos que permitían explicar mejor las relaciones entre la calidad del servicio online y la satisfacción o la intención de nueva compra, y evitaron centrarse en los aspectos técnicos del diseño y de la calidad del servicio (como las fuentes, los colores, el número de clics, etc.). Su modelo daba cuenta de cuatro dimensiones sobre la calidad de servicio (diseño del sitio web, servicio al cliente, garantía y gestión de los pedidos) y de dos dimensiones sobre la relación (satisfacción y fidelidad hacia el sitio web).

Ivyanno y Nila (2013) examinaron la influencia de la calidad del servicio y la satisfacción del turista en las futuras intenciones de comportamiento. Para ello, utilizaron el modelo SERVQUAL y el análisis de regresión múltiple. El estudio probó que la calidad del servicio tiene una influencia positiva en la satisfacción de los usuarios.

De las investigaciones anteriores, parece deducirse que las dimensiones que mejor definen la calidad de servicio en un sitio web de viajes son la experiencia de compra, el diseño, la seguridad, la información y la comunicación.

Concretamente, el estudio realizado por Wen (2009), afirma que la experiencia que los usuarios desarrollan en los sitios web de viajes a través del e-commerce, repercute en la satisfacción de los clientes. Otros estudios como el de Rheem (2010) afirman que el aumento de las opciones a la hora de comprar algún producto en un sitio web de viajes tiene un impacto sobre la satisfacción de los usuarios y sobre el comportamiento de compra. Por ello, parece plausible formular la hipótesis siguiente:

$\mathrm{H}_{1}$ La experiencia de compra en el sitio web tiene un efecto positivo en la satisfacción online.

El diseño de los sitios web de viajes juega un papel muy importante en el desarrollo de la actitud y el comportamiento hacia la marca. Las características del sitio web determinan la percepción de los usuarios hacia la tienda online. Un buen diseño es fundamental para localizar la información y reduce el tiempo de búsqueda de información (Luo, Ba y Zhang, 2012). En efecto, el estudio realizado por Wen (2009) concluye que un buen diseño del sitio web de viajes incrementa la satisfacción de los usuarios (Qi, Law y Buhalis, 2008). Por ello, formulamos la siguiente hipótesis:

$\mathrm{H}_{2}$ El diseño del sitio web tiene un efecto positivo en la satisfacción online.

La seguridad es definida como la capacidad que los sitios web de viajes tienen para proteger la información personal de los usuarios durante el proceso de transacciones o de intercambio de información. Algunos estudios como el Chen y Dibb (2010) demuestran que el buen manejo en la información de los usuarios tiene un efecto positivo en la satisfacción. Esta percepción de seguridad en los sitios web de viajes, nos lleva a platearnos la tercera hipótesis:

$\mathrm{H}_{3}$ La seguridad del sitio web tiene un efecto positivo en la satisfacción online.

La información ofrecida en un sitio web no solo ha de evaluarse por su relevancia, sino también por su calidad y por su accesibilidad (Lin, 2010). Autores como Sindhuja y Dasticar (2009) argumentan que el contenido informativo es un indicador relevante para 
determinar la satisfacción de los usuarios. El contenido de información tiene un impacto directo sobre los usuarios y sobre su evaluación de la eficacia del sitio web (Salavati y Hashim, 2015). Por ello, los sitios web deben presentar información suficiente y relacionada con la descripción del producto, sobre los precios y los servicios suplementarios con la finalidad de ayudar a los usuarios. El trabajo de investigación de Wen (2009) demuestra que, si los usuarios encuentran un sitio web adecuado, desarrollarán sentimientos positivos hacia el sitio web y estarán más satisfechos. Ante este contexto, plateamos la cuarta hipótesis:

$\mathrm{H}_{4}$ La información del sitio web tiene un efecto positivo en la satisfacción online.

Los sitios web de viajes son el primer punto de contacto entre los proveedores y los usuarios. Por ello, es vital importancia considerar la comunicación como una dimensión principal de la calidad de servicio online (Rimmington and Yuksel, 1998). El trabajo realizado por Latiff y Siew Imm (2015) demuestra la relación entre la comunicación y la satisfacción de los usuarios de los sitios web de viajes. Por ello, parece correcto formular la hipótesis siguiente:

$\mathrm{H}_{5}$ La comunicación del sitio web tiene un efecto positivo en la satisfacción online.

\section{METODOLOGÍA}

La metodología empleada en la investigación para la consecución de los objetivos y la constatación de las hipótesis propuestas se basa en técnicas cualitativas y cuantitativas. Malhotra (2008) y Hair et al. (2010) afirman que la investigación cualitativa se utiliza para identificar las dimensiones de estudio; en nuestro caso, la hemos empleado para identificar las dimensiones que definen mejor la calidad de servicio. En cambio, la investigación cuantitativa la desarrollamos para analizar la relación entre las dimensiones (Malhotra, 2008; Hair et al., 2010); en nuestro caso, la influencia de las dimensiones de la calidad de servicio online en la satisfacción de los usuarios de sitios web de viajes.

En el apartado cualitativo, realizamos una reunión de grupo (focus group). Como señalan Malhotra (2008) y Hair et al. (2010), los participantes de un focus group deben contar con conocimientos suficientes sobre el tema a estudiar. Por ello, en nuestra investigación, hemos elegido expertos del sector de sitios web de viajes que podían aportar información subjetiva y válida para la realización de la investigación. Así, los expertos seleccionados (Vallespín y Molinillo, 2014, p. 17) fueron los siguientes: (1) académicos cuya línea de investigación está relacionada con la calidad de servicio online en el sector turístico y que fueron identificados por su contribución a la bibliografía; (2) profesionales del ámbito turístico que representan a la mayoría de los sectores: directores de empresas de servicios turísticos (hoteles y empresas de transporte fundamentalmente), directores de empresas intermediarias (AAVV y TTOO) y directores de empresas de soluciones tecnológicas cuyos clientes son las empresas turísticas; y (3) responsables máximos de la administración cuyo cargo les permitía poseer una visión amplia sobre qué es lo que está ocurriendo en el sector y sobre qué puede ocurrir en un futuro próximo en los sitios web de viajes (Sarmiento, 2015; de Esteban Curiel, 2007; Antonovica, 2012). 
Según Malhotra (2008) y Hair et al. (2010), para desarrollar correctamente un focus group, es necesario una muestra de 8 a 12 participantes; en nuestra investigación la muestra está formada por 10 personas ( 5 hombres y 5 mujeres) con edades comprendidas entre los 25 y 65 años. Para el grupo, se utilizó la herramienta de la entrevista de forma no estructurada y natural en la que un moderador (en este caso el investigador) guiaba la discusión para obtener información al escuchar a los grupos de personas del mercado meta y para llegar al principal objetivo de esta primera fase. A los participantes se les obsequió con un objeto para que contestaran de forma objetiva. También, según señalan Malhotra (2008) y Hair et al. (2010), en este tipo de investigaciones se han de realizar dos sesiones como mínimo y hay que proseguirlas hasta que los grupos entrevistados no ofrezcan ya más ideas u opiniones. En nuestra investigación, se utilizaron dos sesiones; en la principal se presentaba el tema a los participantes y en la de cierre se resumían todos los aspectos más importantes.

En el apartado cuantitativo, se optó por usuarios que frecuentaran los sitios web de viajes regularmente con el principal objetivo de poder analizar las dimensiones que forman la calidad de servicio online y su influencia en la satisfacción de los usuarios. En este contexto, elegimos a los jóvenes universitarios de la Comunidad de Madrid como población de nuestro estudio. Lo importante no era investigar si los jóvenes universitarios sabían utilizar los diferentes sitios web de viajes. Importaba más obtener unidades de análisis que poseyeran la suficiente experiencia acumulada para poder estudiar la calidad del servicio ofrecido y su influencia en la satisfacción del usuario. Y fue también la razón principal por la que se eligió una población de jóvenes universitarios con edades comprendidas entre 18 y 24 años; porque disfrutaban de una media de 4 horas y 42 minutos al día en Internet, lo que proporcionaba una experiencia acumulada relevante para nuestro estudio (Nielsen, 2015; Sánchez Franco et al., 2012). Por limitaciones como la dificultad de acceso y por el coste, hemos elegido la técnica del muestreo estratificado y el muestreo aleatorio simple.

Respecto al muestreo estratificado, decidimos dividir la población en estratos siguiendo un criterio relacionado con el objeto de nuestro estudio. En nuestro caso, dividimos los jóvenes universitarios de la Comunidad de Madrid en dos estratos: universidades públicas (Universidad Rey Juan Carlos) y universidades privadas (Centro universitario ESERP). Como afirma De Esteban Curiel (2007), para llevar a cabo este método, la asignación del tamaño de la muestra de cada estrato admite diversas posibilidades; la más utilizada y la elegida para la investigación es la asignación proporcional en donde la proporción de los elementos de la muestra pertenecientes a un estrato presenta la misma proporción que los elementos de la población de ese estrato. 
Tabla 3. Ítems utilizados para evaluar la calidad del servicio online y la satisfacción online

\begin{tabular}{|c|c|c|}
\hline ítem & Abreviatura & Referencia \\
\hline \multicolumn{3}{|l|}{ Experiencia } \\
\hline Este sitio web de viajes es muy cómodo para comprar & EXP1 & \multirow{3}{*}{$\begin{array}{l}\text { Chung y Shin } \\
(2010)\end{array}$} \\
\hline Este sitio web facilita buenos productos & EXP2 & \\
\hline En este sitio me siento seguro para realizar compras & EXP3 & \\
\hline \multicolumn{3}{|l|}{ Diseño } \\
\hline El sitio web es vialmente atractivo & DS1 & \multirow{3}{*}{$\begin{array}{l}\text { Cristóbal et } \\
\text { al. (2007) }\end{array}$} \\
\hline El sitio web tiene una buena selección de servicios & DS2 & \\
\hline El sitio web tiene una apariencia profesional & DS3 & \\
\hline \multicolumn{3}{|l|}{ Seguridad } \\
\hline Este sitio web protege la privacidad de los usuarios & SG1 & \multirow{3}{*}{$\begin{array}{l}\text { Parasuraman } \\
\text { et al. (2005) }\end{array}$} \\
\hline Este sitio web no utilizará mi información personal & SG2 & \\
\hline Este sitio web no comercializará con información personal & SG3 & \\
\hline \multicolumn{3}{|l|}{ Información } \\
\hline Este sitio web proporciona información de calidad de los viajes & INF1 & \multirow{3}{*}{$\begin{array}{l}\text { Kim et al., } \\
(2009)\end{array}$} \\
\hline Este sitio web proporciona información precisa de los viajes & INF2 & \\
\hline Este sitio web tiene enlaces a otros sitios web de viajes con más información & INF3 & \\
\hline \multicolumn{3}{|l|}{ Comunicación } \\
\hline Los usuarios pueden poner libremente un comentario en este sitio web & COM1 & \multirow{3}{*}{$\begin{array}{l}\text { Zeithaml et } \\
\text { al. (2013) }\end{array}$} \\
\hline Este sitio web dispone de otros sitios web para comunicarse & COM2 & \\
\hline Este sitio web ofrece vídeos de viaje con posibilidad de comentarlos & СOM3 & \\
\hline \multicolumn{3}{|l|}{ Satisfacción } \\
\hline Yo estoy muy satisfecho con lo que ofrece el sitio web & STF1 & \multirow{3}{*}{$\begin{array}{l}\text { Chung y Shin } \\
(2010)\end{array}$} \\
\hline Yo estoy muy satisfecho con los comentarios de este sitio web & STF2 & \\
\hline Yo estoy muy satisfecho con la profesionalidad del sitio web de viajes & STF3 & \\
\hline
\end{tabular}
Elaboración propia.

Respecto del muestreo aleatorio, hemos decidido seleccionar sistemáticamente cada elemento de la muestra según la población de cada estrato. En el caso de la Universidad de ESERP, necesitábamos una muestra de 82 unidades; la Universidad tiene una población de 1500 alumnos. Si dividimos la población $N$ entre la muestra $n$, nos da el intervalo $K=18$; es decir, de cada 20 personas que entraban en la Biblioteca se seleccionaba una hasta llegar a 82 unidades. En el caso de la Universidad Rey Juan Carlos, la población es de 35.476 alumnos y la muestra es de 494 unidades, lo que da un intervalo de $\mathrm{K}=71$; es decir, de cada 71 personas que entraban en la Biblioteca se seleccionaba una hasta llegar a 494 unidades. Importa añadir que el momento de realización de la encuesta eran fechas de exámenes, lo que aumentó en un porcentaje bastante elevado el número de unidades.

En total, la muestra fue de 576 usuarios encuestados con un error de un 6,7\% para un nivel de confianza del 95\%. Previamente se comprobó la adecuación del cuestionario a través de un pre-test basado en un número reducido de encuestados (30 personas) que se realizó entre los meses de mayo y junio de 2016. El método utilizado fue el aleatorio simple.

Para la recogida de la información, se utilizó la técnica de la encuesta autoadministrada y el cuestionario como herramienta. El cuestionario estaba formado por 27 preguntas relativas a los diferentes sitios web turísticos de la clasificación presentada. Tenía tres apartados: el primero consistía en dos preguntas filtro para dar mayor validez a nuestro trabajo de investigación; el segundo constaba de dieciocho preguntas relacionadas con las dimensiones de la calidad del servicio online y la satisfacción de los sitios web turísticos; y el último eran cuatro preguntas socio-demográficas. Para nuestro cuestionario, se utilizaron preguntas estructuradas múltiples, dicotómicas y de escala. Y, para la medición de preguntas de escala, se utilizó la de Likert de 5 puntos, donde 1 indicaba que el encuestado estaba muy Investigaciones Turísticas 
en desacuerdo y 5 , que estaba muy de acuerdo. Para el análisis e interpretación de los resultados, se utilizó el SPSS (distribución de frecuencias, promedios, medidas de dispersión). En la tabla 3 se muestran los atributos incluidos en la investigación y cuya elección tuvo en cuenta la revisión bibliográfica citada con anterioridad.

\section{ANÁLISIS Y RESULTADOS}

En este apartado, se analizan y presentan los resultados de los datos obtenidos de las 576 encuestas realizadas a los usuarios de los sitios web de servicios turísticos (sitios web de transportes, alojamientos, de opinión, meta-buscadores, de destinos y agencias de viajes). Los porcentajes ofrecidos en las siguientes tablas están calculados en función del tamaño de la muestra de 576 encuestas a través del muestreo aleatorio simple. Este método ha permitido obtener una muestra que, tanto en tamaño como en forma, es representativa de la sociedad objeto de estudio.

En la tabla 4, presentamos la demografía de los usuarios encuestados; para ello, utilizamos un análisis descriptivo de frecuencias. En la pregunta número uno, se les preguntaba por el sexo, el $31,1 \%$ de los encuestados eran varones y el $68,9 \%$, mujeres. Respecto de las edades de los usuarios, el 26,2\% de los encuestados tenía entre 18-30 años; el $46,9 \%$, entre 30 y 50 años siendo la muestra más representativa; el $17,5 \%$ de los encuestados tenía entre 50-60 años; y, por último, el 9,4\% de los encuestados tenía más de 65 años. Respecto del nivel de estudios, la mayoría de los encuestados, un 56,59\% eran graduados; un 10,95\% tenía bachillerato; un 25,87\%, un máster; y un 6,59\% eran doctores. La mayoría de los encuestados tenía la nacionalidad española: un 90,1\% eran españoles, y un 9,9\% eran extranjeros. Las nacionalidades extranjeras eran la china, rumana, británica, francesa, italiana, colombiana, venezolana, ecuatoriana y alemana.

Tabla 4. Demografía de los usuarios de los sitios web de viajes

\begin{tabular}{cccc}
\hline Variables & Categoría & Frecuencia & Porcentaje (\%) \\
\hline Sexo & Masculino & 179 & 31,1 \\
& Femenino & 397 & 68,9 \\
Edad & $18-30$ años & 151 & 26,2 \\
& $30-50$ años & 270 & 46,9 \\
& $50-65$ años & 101 & 17,5 \\
& 65 o más años & 54 & 9,4 \\
Estudios & Bachillerato & 63 & 10,95 \\
& Graduado & 326 & 56,59 \\
& Máster & 149 & 25,87 \\
& Doctor & 38 & 6,59 \\
Nacionalidad & Español & 519 & 90,1 \\
& Extranjero & 57 & 9,9 \\
\hline
\end{tabular}

Elaboración propia.

En las entrevistas en grupo, utilizamos un análisis de contenido que consiste en el análisis sistemático en el que se toman las respuestas y se agrupan en categorías temáticas o pautas (Malhotra, 2008; Hair et al., 2010). En la investigación, para identificar las dimensiones más idóneas para medir la calidad de servicio online, se recurrió a cinco categorías temáticas, que son la experiencia de compra, el diseño, la seguridad, la información y la comunicación. Los resultados indican que 8 de los 10 (80\% de los resultados) expertos identificaron la compra como una de las principales razones por las que 
muchos usuarios disfrutan de la experiencia online. Respecto al diseño, 7 de los 10 (70\% de los resultados) expertos afirmaron que los sitios web de viajes sustituyen a la tienda física, por lo que puede llegar a tener un efecto positivo en la calidad de servicio online. La seguridad en las transacciones y la privacidad de los usuarios fueron considerados por 8 de los 10 (80\% de los resultados) expertos como dimensiones fundamentales para que los usuarios desarrollen una confianza hacia los sitios web de viajes. La información disponible en los sitios web sobre los productos y servicios turísticos es lo que más atrae a los usuarios; en este caso, 9 de los 10 ( $90 \%$ de los resultados) expertos lo consideraron fundamental. Por último, la comunicación entre el sitio web de viajes y los usuarios se considera como el factor más importante; los expertos afirmaron unánimemente que hoy en día para considerar un sitio web de calidad se requiere disponer de herramientas interactivas para el desarrollo de relaciones de calidad.

En la figura 1, podemos apreciar que los sitios web de viajes más utilizados son los motores de búsqueda con un 41\%; es decir, 236 usuarios afirmaron utilizar este tipo de sitio web. Por el contrario, el menos utilizado es el sitio web de destinos turísticos con un $0,70 \%$; es decir, 4 usuarios afirmaron haber utilizado este tipo de sitio web. Al realizar el análisis, pudimos constatar que los sitios web de viajes más utilizados son los que disponen de herramientas interactivas, es decir, de medios sociales para poder intercambiar contenido.

Figura 1: Uso de los sitios web de viajes

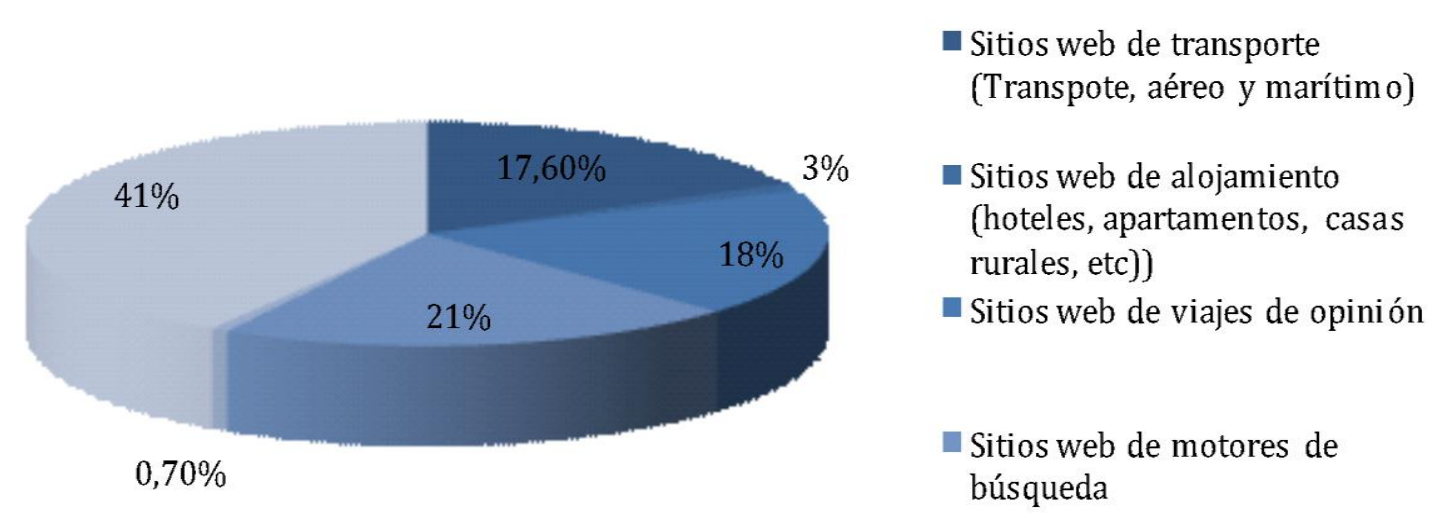

Elaboración propia.

Respecto del apartado cuantitativo, el cuestionario fue sometido a un análisis factorial confirmatorio donde todas las cargas y valores " $\mathrm{t}$ " significativos fueron menores a un valor $p<.01$. En la tabla 5 , se describe el valor de la media y las desviaciones estándar para cada variable en el cuestionario de estudio. Los resultados mostraron que la compra en el sitio web de viajes $(3,99)$ y la comunicación $(3,92)$ son las medias más altas, lo que muestra su importancia desde la percepción de los usuarios de la muestra, mientras que la media del diseño es la más baja $(3,58)$, lo que indica que los usuarios de los sitios web de viajes consideran que es menos importante. La satisfacción de los usuarios como variable dependiente se expresó en una media de 3,67 , lo que está considerado como una satisfacción aceptable. Para medir la fiabilidad del cuestionario utilizado, se aplicó el alfa de Cronbach, obteniéndose valores superiores a 0,60 como indica Hair et al., (2010). En segundo lugar, se utilizó un análisis de fiabilidad compuesta en la que se obtuvieron resultados superiores a 0,70, como también indican Hair et al., (2010). Por último, se 
recurrió a un análisis de la varianza extraída promedio, donde los resultados fueron superiores a .50 (Hair et al., 2010). En la tabla 5, se presentan todos los resultados del análisis factorial confirmatorio.

Tabla 5. Análisis factorial confirmatorio

\begin{tabular}{lllll}
\hline Dimensión & $\begin{array}{l}\text { Media/ } \\
\text { Desviación }\end{array}$ & $\begin{array}{l}\text { Alfa de } \\
\text { Cronbach }\end{array}$ & AVE & $\begin{array}{l}\text { Fiabilidad } \\
\text { Compuesta }\end{array}$ \\
\hline Experiencia & $3,99 / 0.793$ & 0,893 & 0,92 & 0,98 \\
Diseño & $3,58 / 0.903$ & 0,723 & 0,93 & 0,97 \\
Seguridad & $3,63 / 0.996$ & 0,789 & 0,94 & 0,91 \\
Información & $3,85 / 0.847$ & 0,834 & 0,90 & 0,93 \\
Comunicación & $3,92 / 0.786$ & 0,912 & 0,93 & 0,93 \\
Satisfacción & $3,67 / 0.994$ & 0,865 & 0,94 & 0,95 \\
\hline
\end{tabular}

Elaboración propia.

Una vez demostrada la fiabilidad del instrumento, recurrimos a la validez del mismo. Para ello, utilizamos la validez discriminante que mide el grado en que una determinada dimensión es diferentes del resto. En la tabla 6, se puede observar que ninguno de los valores de confianza incluye el valor 1 . Así, podemos concluir que los factores utilizados en el modelo tienen una fiabilidad y validez adecuadas.

Tabla 6. Resultados de la validez discriminante

\begin{tabular}{lllllll}
\hline & 1 & 2 & 3 & 4 & 5 & 6 \\
\hline 1 Experiencia & $\mathbf{0 , 8 5 4}$ & & & & & \\
2 Diseño & 0,367 & $\mathbf{0 , 6 1 3}$ & & & & \\
3 Seguridad & 0,381 & 0,345 & $\mathbf{0 , 7 7 8}$ & & & \\
4 Información & 0,467 & 0,374 & 0,445 & $\mathbf{0 , 7 9 8}$ & & \\
5 Comunicación & 0,456 & 0,376 & 0,365 & 0,367 & $\mathbf{0 , 8 4 5}$ & \\
6 Satisfacción & 0,367 & 0,445 & 0,386 & 0,467 & 0,423 & $\mathbf{0 , 7 1 2}$ \\
\hline
\end{tabular}

Elaboración propia.

En la tabla 7, se presenta la constatación de las hipótesis con un modelo de ecuaciones estructurales mediante el método de máxima verosimilitud (Hair et al., 2010). Como se puede observar, existe una relación positiva entre la compra en sitio web de viajes y la satisfacción de los usuarios $(t=5,057, p=, 000)$. En consecuencia, se acepta la hipótesis $H_{1}$. Además, el estudio mostró una relación positiva entre el diseño de los sitios web de viajes y la satisfacción de los usuarios ( $t=5,285 ; p=, 000)$, lo que nos legitima para aceptar la hipótesis $\mathrm{H}_{2}$. Los resultados también mostraron que la seguridad en los sitios web de viajes es una dimensión relevante en la satisfacción de los usuarios $(t=3,319 ; p=, 001)$. Por consiguiente, se acepta la hipótesis $\mathrm{H}_{3}$. De la hipótesis $\mathrm{H}_{4}$ sobre la relación positiva entre la información y la satisfacción de los usuarios, los resultaron prueban esa relación ( $t=6,232 ; p=$ ,000). Por último, en los resultados se puede observar que existe una relación positiva entre la comunicación y la satisfacción de los usuarios ( $\mathrm{t}=2.496 ; \mathrm{p}=0.01$ ), por lo que se acepta la hipótesis H5. La $R^{2}$ de 0,664 mostró que el modelo se explica en un $66 \%$; es decir, la satisfacción de los usuarios se explica en un $66 \%$ mediante la compra, el diseño, la seguridad, la información y la comunicación de los sitios web de viajes. El valor $\mathrm{p}$ para el modelo fue menor a 0,05 lo que indica que se pueden aceptar las hipótesis planteadas y que al menos alguno de los parámetros es distinto de cero; por consiguiente, el modelo es válido en su conjunto. 
Tabla 7. Modelo estructural propuesto para analizar la calidad de servicio online y su influencia en la satisfacción de los usuarios en los sitios web de viajes

\begin{tabular}{|c|c|c|c|c|}
\hline Relación estructural & B est & rizados & Valor $\mathrm{t}$ & Criterio \\
\hline $\begin{array}{l}\mathrm{H}_{1} \text { La experiencia de compra en el sitio } \\
\text { web tiene un efecto positivo en la } \\
\text { satisfacción online. }\end{array}$ & 202 & $*$ & 5,057 & Se acepta \\
\hline $\begin{array}{l}\mathrm{H}_{2} \text { El diseño del sitio web tiene un efecto } \\
\text { positivo en la satisfacción online. }\end{array}$ & 205 & $*$ & 5,285 & Se acepta \\
\hline $\begin{array}{l}\mathrm{H}_{3} \text { La seguridad del sitio web tiene un } \\
\text { efecto positivo en la satisfacción online. }\end{array}$ & 132 & $*$ & 3,319 & Se acepta \\
\hline $\begin{array}{l}\mathrm{H}_{4} \text { La información del sitio web tiene un } \\
\text { efecto positivo en la satisfacción online. }\end{array}$ & 247 & $*$ & 6,232 & Se acepta \\
\hline $\begin{array}{l}\mathrm{H}_{5} \text { La comunicación del sitio web tiene un } \\
\text { efecto positivo en la satisfacción online. }\end{array}$ & 145 & $*$ & 2,496 & Se acepta \\
\hline
\end{tabular}

Nota. $\mathrm{N}=576 ;{ }^{*} \mathrm{p}>0,01 ; \mathrm{R}^{2}=0,664 ; \mathrm{x} 2(\mathrm{p})=44,844(0,000) ; \mathrm{NNFI}=0,923 ; \mathrm{CFI}=0,943 ; \mathrm{IFI}=0,975 ; \mathrm{RMSA}=0,078$ Elaboración propia.

Todos estos resultados vienen a corroborar la relación existente entre la calidad del servicio online y la satisfacción de los usuarios, como bien argumentaron Cristóbal et al. (2007), Liu et al. (2008), Herington y Weaven (2009) y Kim et al. (2009). En esta investigación, para comprobar el ajuste del modelo recurrimos al Goodness of Fit Index (GFI), al Normed Fit Index (NFI) y al Comparative Fit Index (CFI) en los que obtuvimos valores cercanos a 1, como recomiendan Hair et al. (2010). Por último, utilizamos, el Root Mean Square Error of Aproximation (RMSEA) y se obtuvo un valor cercano a 0,08, como también recomienda Hair et al. (2010).

\section{CONCLUSIONES}

En este apartado, recogemos las conclusiones en el mismo orden de las preguntas. En efecto, las contribuciones empíricas más importantes a la praxis académica han sido las siguientes:

Sobre el objetivo general planteado al principio de nuestra de investigación, podemos afirmar que existe una relación entre las dimensiones (experiencia de compra, diseño, seguridad, información y comunicación) que conforman la calidad de servicio en los sitios web de viajes y la satisfacción de los usuarios. Podemos concluir que las dimensiones que más valoran los usuarios son, en primer lugar, la experiencia de compra y, en segundo, la información que se les proporciona para la planificación de sus viajes. La dimensión que menos valoran los usuarios es la comunicación, ya que la mayoría de los sitios web de viajes no pone a disposición de los usuarios herramientas para desarrollar la interactividad.

Del primer objetivo específico sobre las dimensiones de la calidad de servicio, podemos afirmar que, después de haber realizado una revisión de la bibliografía académica sobre este tema y un análisis mediante técnicas cualitativas, la calidad de servicio está 
formada principalmente por las dimensiones siguientes: (1) la experiencia de compra, que es una de las principales razones por las que muchos usuarios disfrutan de la experiencia online; (2) el diseño, que sustituye a la tienda física, por lo que puede llegar a tener un efecto positivo para realizar compras o para buscar información; (3) la seguridad en las transacciones y la privacidad de los usuarios son dos de los factores más importantes de los sitios web; (4) la información disponible en los sitios web sobre los productos y los servicios es un aspecto relevante; y (5) la comunicación entre usuarios, que es muy importante para construir comunidades online y coproducir el servicio; por consiguiente, facilitar herramientas interactivas para poder comunicarse resulta esencial.

En relación con el segundo objetivo específico, podemos concluir que la calidad de servicio online se define como el grado en que un sitio web facilita la experiencia de compra y la interactividad entre los usuarios. Los sitios web de viajes han de entenderse como tiendas virtuales en donde los usuarios puedan desarrollar relaciones de calidad con la empresa que les presta el servicio.

Del tercer objetivo sobre la identificación y la clasificación de los diferentes tipos de sitios web de viajes que podemos encontrar en el contexto digital, hemos constatado, tras haber realizado una revisión de la literatura existente sobre el tema y una investigación mediante técnicas cualitativas, que existen seis tipos de sitios web de viajes:

- Los sitios web de alojamiento que están constituidos por hoteles, hostales, balnearios, campings, casas rurales, etc. Su principal actividad es proporcionar a todos los usuarios alojamiento.

- Los sitios web de transporte que están formados por el transporte aéreo, marítimo o terrestre. Su principal actividad es ofrecer cualquier tipo de transporte a los usuarios.

- Los sitios web de agencias de viajes centrados en ofrecer a los usuarios paquetes turísticos (alojamiento, transporte, servicios, etc.) para ahorrarles entrar en varios sitios web para planificar sus viajes.

- Los sitios web de motores de búsqueda ideados principalmente para ofrecer a los usuarios el mejor precio del mercado. Esto se logra utilizando un algoritmo que rastrea todos los sitios web de viajes y localiza el mejor precio.

- Los sitios web de destinos suelen ser institucionales y se centran en ofrecer a los usuarios toda la información necesaria sobre una ciudad, país o destino. Son los sitios web de viajes menos utilizados, por ser poco atractivos y por no desarrollar la interactividad entre usuarios.

- Los sitios web de opinión son plataformas en las que los usuarios constituyen el centro del negocio por ser ellos quienes opinan y generan contenido (fotografías y vídeos). Por ello, el sitio web de viajes más atractivo para los usuarios con diferencia, y que destaca entre ellos, es Tripadvisor.

Permítasenos terminar, aunque no es un objetivo de este trabajo de investigación, con los resultados sobre el uso de los sitios web de viajes. El $41 \%$ de los usuarios utiliza los sitios web de las agencias de viajes y es el resultado el más significativo. El 0,7\% utiliza los sitios web de destinos; el 21,1\%, los sitios web de motores de búsqueda; el 18,5\%, los sitios web de viajes de opinión; el 1,1\%, los sitios web de alojamientos (hoteles, moteles, hostales, 
casas rurales, etc.); y, por último, el 17,6\% utiliza los sitios web de proveedores de transporte (aéreo, marítimo y terrestre).

\subsection{Implicaciones}

La calidad de servicio en un sitio web de viajes puede reforzar las actitudes de los usuarios hacia las marcas. El modelo propuesto en este trabajo de investigación, demuestra que las dimensiones de la experiencia, diseño, seguridad, información y comunicación influyen positivamente en su satisfacción. En este estudio, las mediciones de la satisfacción online de los usuarios tenían una respuesta emocional directa a través de la experiencia con los distintos sitios web analizados. Una de las principales dimensiones de estudio en esta investigación es la determinación de si el diseño es un factor importante para indicar la satisfacción de los usuarios, ya que los resultados muestran una influencia sustancial, pero no determinante. Por otro lado, las dimensiones de la información y comunicación vienen a probar que las organizaciones de viajes online tienen que desarrollar relaciones con los usuarios mediante la interactividad y el desarrollo de vínculos sociales, pues tienen efectos muy positivos sobre la satisfacción y el patrocinio de los usuarios a través de los medios sociales.

\subsection{Limitaciones y futuras líneas de investigación}

En el modelo propuesto en esta investigación se han identificado dos limitaciones. La primera de ellas es que esta investigación solo se centra en la Comunidad de Madrid, excluyendo a las otras comunidades de España. La segunda limitación es que el estudio se centra en jóvenes universitarios, excluyendo a otros usuarios no universitarios y de rangos de edad diferentes.

Para futuras investigaciones, consideramos conveniente incorporar a este modelo las dimensiones de confianza y compromiso como consecuentes de la calidad de servicio en los sitios web de viajes. Las dimensiones de confianza, satisfacción y compromiso forman lo que se denomina como la calidad de relación y son lo que desarrolla una actitud positiva hacia el sitio web de viajes. Otro aspecto interesante sería analizar la influencia de la calidad de servicio online sobre el comportamiento de compra, la fidelización o la comunicación de boca en boca. Además, sería muy interesante analizar la fidelización o comunicación de boca en boca como consecuentes de la calidad de servicio.

\section{REFERENCIAS BIBLIOGRÁFICAS}

Anderson, E. W., Fornell, C., y Lehman, D. R. (1994). Customer Satisfaction, Market Share and Profitability: Findings from Sweden. Journal of Marketing, 58 (3), 53-66.

Anderson, R. E., y Srinivasan, S. S. (2003). E-satisfaction and e-loyalty: A contingency framework. Psychology and Marketing, 20, 123-138.

Antonovica, A. (2012). Comunicación e imagen de los países bálticos en España a través de la técnica del discurso periodístico (Vol. 156). Editorial Dykinson.

Athanasopoulou, P. (2009). Relationship quality: A critical literature review and research agenda. European Journal of Marketing , 43 (5/6), 583-610.

Bansal, H. S., McDougall, G. H. G., Dikolli, S. S. y Sedatole, K. L. (2004). Relating e-Satisfaction to Behavioral Outcomes: an Empirical Study. Journal of Services Marketing, 18 (4), 290302. 
Barnes, S. y Vidgen, R. (2002). An integrative Approach to the Assessment of E-Commerce. Journal of Electronic Commerce Research, 3 (3), 114-126.

Bauer, H. H., Falk, T. y Hammerschmidt, M. (2006). A transaction process-based approach for capturing service quality in online shopping. Journal of Business Research, 59, 866-875.

Boyd, H.W., Walker, O. C., Mullins, J. y Larreche J. (2002). Marketing Management, A Strategic Decision-Making Approach. McGraw-Hill/Irwin, Columbus, $\mathrm{OH}$.

Buhalis, D. y Law, R. (2008). Progress in information technology and tourism management: 20 year on and 10 years after the internet: the state of e-Tourism research. Tourism Management, 29 (4), 609-623.

Casaló, L. V., Flavian, C. y Guinalíu, M. (2008). The Role of Satisfaction and Website Usability in Developing Customer Loyalty and Positive Word-of-Mouth in the e-Banking Services. The International Journal of Bank Marketing, 26 (6), 399-417.

Chenet, P., Tynan, C., y Money, A. (1999). Service performance gap: Reevaluation and redevelopment. Journal of Business Research, 48, 133-147.

Cho, N. y Park, S. (2001). Development of electronic commerce user-consumer satisfaction index (ECUSI) for Internet shopping. Industrial Management \& Data Systems, 101 (8), 400-406.

Chung, K. H., y Shin, J. I. (2010). The Antecedents and Consequents of Relationship Quality in Internet Shopping. Asia Pacific Journal of Marketing and Logistics, 22 (4), 473-491.

Collier, J. E. y Bienstock, C. C. (2006). Measuring Service Quality in e-Retailing. Journal of Service Research, 8 (3), 260-275.

Connolly, D. J., Olsen, M. D. y Moore, R. G. (1998). The internet as a distribution channel. The Cornell Hotel and Restaurant Administration Quarterly, 39 (4), 42-54.

Constantinides, E. (2004). Influencing the online consumer's behavior: The web experience. Journal of Internet Research, 14(2), 111-126.

Constantinides E. y Fountain S. (2007). Web 2.0: Conceptual Foundations and Marketing Issues. Journal of Direct, Data and Digital Marketing Practice 9 (3).

Cox, J. y Dale, B. G. (2002). Key quality factors in Web site design and use: an examination. The International Journal of Quality \& Reliability Management, 19 (6/7), 862-888.

Cristóbal, E., Flavian, C. y Guinaliu, M. (2007). Perceived e-service quality (PeSQ): Measurement validation and effects on consumer satisfaction and web site loyalty. Managing Service Quality, 17 (3), 317-340.

de Esteban Curiel, J. (2007). La demanda del turismo cultural y su vinculación con el medio ambiente urbano los casos de Madrid y Valencia (Doctoral dissertation, Universidad Complutense de Madrid).

Devaraj, S., Fan, M. y Kohli, R. (2002). Antecedents of B2C channel satisfaction and preference: validating e-Commerce metrics. Information System Research, 13 (3), 31633.

Egan, J. (2011). Relationship Marketing: Exploring Relational Strategies in Marketing. Financial Times / Prentice Hall.

Elliott, M. T. y Speck, P. S. (2005). Factors that affect attitude toward a retail web site. Journal of Marketing Theory and Practice, 13 (1), 40-51.

Ennew, C. T. y Binks, M. R. (1999). The Impact of Participative Service Relationships on Quality, Satisfaction and Retention: An Exploratory Study. Journal of Business Research, 46, 121-132. 
Evanschitzky, H., Iyer, G. R., Hesse, J. y Ahlert, D. (2004). E-Satisfaction: a re-examination. Journal of Retailing, 80 (3), 239-247.

Fassnacht, M. y Koese, I. (2006). Quality of Electronic Services: Conceptualizing and Testing a Hierarchical Model. Journal of Service Research, 9 (1), 19-37.

Fassnacht, M., Köse, I. (2007). Consequences of Web-based service quality: uncovering a multifaceted chain of effects. Journal of Interactive Marketing, 21 (3), 35-54.

Francis, J. E., y White, L. (2002). Exploratory and confirmatory factor analysis of the perceived internet retailing quality (PIRQUAL) model. In R. N. Shaw, S. Adam, \& H. McDonald (Eds.), Proceedings of ANZMAC.

Gefen, D. (2002). Reflections on the dimensions of trust and trustworthiness among online consumers. ACM Sigmis Database, 33(3), 38-53.

Gounaris, S., Dimitriadis, S. y Stathakopoulos, V. (2010). An Examination of the Effects of Service Quality and Satisfaction on Customers' Behavioral Intentions in E-shopping. Journal of Services Marketing, 24 (2), 142-156.

Gretzel, U. (2006). Consumer generated content-trends and implications for branding. EReview of Tourism Research, 4 (3), 9-11.

Gummerus, J., Liljander, V., Pura, M. y Van Riel, A. (2004). Customer loyalty to content-based Web sites: the case of an online health-care service. Journal of Services Marketing, 18 (3), 175-186.

Hallowell, R. (1996). The relationships of customer satisfaction, customer loyalty and profitability: an empirical study. International Journal of Service Industry Management, 7 (4), 27-42.

Heiner, E., Gopalkrishnan, R. I., Josef, H. y Dieter, A. (2004). E-satisfaction: a re-examination", Journal of Retailing, 80 (3), 239-47.

Herington, C. y Weaven, S. (2008). Can banks improve customer relationships with high quality online services? Managing Service Quality, 17 (4), 404-427.

Herington, C. y Weaven, S. (2009). E-retailing by banks: e-Service quality and its importance to customer satisfaction. European Journal of Marketing, 43 (9), 1220-1231

Hess, J. y Story, J. (2005). Trust-based commitment: multidimensional consumer-based relationships. Journal of Consumer Marketing, 22 (6), 313-322.

Ho, C. I. y Lee, Y. L. (2007). The development of an e-Travel service quality scale. Tourism Management, 28 (6), 1434-1449.

Hsuehen, H. (2006). An empirical study of web site quality, customer value, and customer satisfaction based on e-shop. The Business Review, 5 (1), 190-193.

Ibrahim, E. E., Joseph, M. y Ibeh, K. I. N. (2006). Customers' perception of electronic service delivery in the UK retail banking sector. International Journal of Bank Marketing, 24 (7), 475-493.

Janda, S., Trocchia, P.J. y Gwinner, K. P. (2002). Consumer Perceptions of Internet Retail Service Quality. International Journal of Service Industry Management 13 (5), 412-431.

Kasavana, L. K., Knuston, B. J., y Polonowski, S. J. (1997). Netlurking: The future of hospitality Internet Marketing. Journal of Hospitality \& Leisure Marketing, 5 (1), 31-44.

Kassim, N. y Abdullah, N. A., (2010). The effect of perceived service equality dimensions on customer satisfaction, trust, and loyalty in e-Commerce settings: A cross cultural analysis. Asia Pacific Journal of Marketing and Logistics, 22 (3), 351-371

Kim, J. H., Kim, M. J. y Kandampully, J. (2009). Buying environment characteristics in the context of e-Service. European Journal of Marketing, 43 (9/10), 1188-1204. 
Kim, S. y Stoel, L. (2004).Apparel retailers: website quality dimensions and satisfaction. Journal of Retailing and Consumer Services, 11 (2), 109-117.

Kim, S. Y. y Lim, Y. J. (2001). Consumers' perceived importance of and satisfaction with internet shopping. Electronic Markets, 11 (3), 148-154.

Koivumaki, T. (2001). Customer satisfaction and purchasing behavior in a web-based shopping environment. Electronic Markets, 11 (3), 186-192.

Koufaris, M. (2002). Applying the technology acceptance model and flow theory to online consumer behavior. Information Systems Research, 13, 205-223.

Landeta, J. (1999). El método Delphi. Ariel, Barcelona.

Lee, G. y Lin, H. (2005). Customer perceptions of e-Service quality in online shopping. International Journal of Retail \& Distribution Management, 33, 2, 161-176.

Lin, Ch. (2010). Examining e-travel sites: an empirical study in Taiwan. Online Information Review, 34 (2), 205-228.

Liu, C. y Arnett, K. P. (2000). Exploring the factors associated with Web site success in the context of electronic commerce. Information and Management, 38, 23-33.

Liu, Y., Li, Y., Tao, L., y Wang, Y. (2008). Relationship stability, trust and relational risk in marketing channels: Evidence from China. Industrial Marketing Management, 37, 432446.

Loiacono, E. T. (2000). Webqual (tm): a web site quality instrument. University of Georgia.

Madu, C. N. y Madu, A. A. (2002). Dimensions of e-quality. International Journal of Quality \& Reliability Management, 19 (3), 246-258.

Mittal, V., W. T. Ross, \& Patrick M. Baldasare (1998). The Asymmetric Impact of Negative and Positive Attribute-Level Performance on Overall Satisfaction and Repurchase Intentions. Journal of Marketing, 62 (1): 33-47.

Ndubisi, N. O. (2008). Relationship quality antecedents: the Malaysian retail banking perspective. International Journal of Quality \& Reliability Management, 24 (8), 829-845.

Nusair, K. y Kandampully, J. (2008). The antecedents of customer satisfaction with online travel services: a conceptual model. European Business Review, 20 (1), 4-19.

O’Neill, M. A. (2001). Measuring service quality and customer satisfaction", J. Kandampully., C. Mok, \& B. Sparks, (Eds.): Service quality management in hospitality, tourism and leisure, New York: Haworth Hospitality Press.

Ofir, C., y Simonson, I. (2007). The effect of stating expectations on customer satisfaction and shopping experience. Journal of Marketing Research, 44(1), 164-174.

Oliver, R. L. (1981). Measurement and Evaluation of Satisfaction Processes in Retail Settings. Journal of Retailing, 57 (3), 25-48.

Oliver, R. L. (1997). Satisfaction: on a Behavioral Perspective on the Consumer, N.Y: McGrawHill.

Pan, B., Hembrooke, H., Joachims, T., Lorigo, L., Gay, G., y Granka, L. (2007). In Google we trust: users' decisions on rank, position and relevancy. Journal of Computer-Mediated Communication, 12 (3), 801-823.

Parasuraman, A. y Zinkhan, G. (2002). Marketing to and serving customers through the Internet: an overview and research agenda. Journal of the Academy of Marketing Science, 30 (4), 286-295.

Parasuraman, A., Zeithaml, V. A. y Malhotra, A. (2005). E-S-QUAL. A Multiple-Item Scale for Assesing Electronic Service Quality. Journal of Service Research, 7 (3), 213-233. 
Park, C. H. y Kim, Y. G. (2003). Identifying key factors affecting consumer purchase behavior in an online shopping context. International Journal of Retail \& Distribution Management, 31 (1), 16-29.

Pudliner, B. A. (2007). Alternative literature and tourist experience: travel and tourist Weblogs. Journal of Tourism and Cultural Change, 5 (1), 46-59.

Ranganathan, C. y Ganapathy, S. (2002). Key dimensions of business-to-consumer web site. Information \& Management, 39 (6), 457-465.

Roberts, R. Varki, S. y R. Brodie (2003). Measuring the quality of relationships in consumer services: An empirical study. European Journal of Marketing, 37 (1/2), 169-196.

Santos, J. (2003). E-service quality: a model of virtual service quality dimensions. Managing Service Quality, 13 (3), 233-246.

Sarmiento Guede, J. R. (2015). Marketing de relaciones. Aproximación a las relaciones virtuales. Dykinson. Madrid.

Sarmiento Guede, J. R. (2016). El impacto de los medios sociales en la estructura del sistema de distribución turístico: análisis y clasificación de los nuevos proveedores de servicios turísticos en el entorno online. Cuadernos de Turismo, (38), 459-483.

Schaupp, L. C. y Belanger, F. (2005). A conjoint analysis of online consumer satisfaction. Journal of Electronic Commerce Research, 6 (2), 95-111.

Shachaf, P., Oltmann, S. M. y Horowitz, S. M. (2008). Service equality in virtual reference. Journal of the American Society for Information Science and Technology, 59 (4), 535-550.

Shamdasani, P., Mukherjee, A. y Malhotra, N. (2008). Antecedents and consequences of service quality in consumer evaluation of self-service internet technologies. The Service Industries Journal, 28 (1), 117-138.

Shankar, V., Amy, K. y Rangaswamy, A. (2003). Customer satisfaction and loyalty in online and offline environments. International Journal of Research in Marketing, 20 (2), 153175.

Sheng, T., y Liu, C. (2010). An empirical study on the effect of e-service quality on online customer satisfaction and loyalty. Nankai Business Review, 1 (3), 273-283.

Shun, C. y Yunjie, X. (2006). Effects of outcome, process and shopping enjoyment on online consumer behavior. Electronic Commerce Research and Applications, 5 (4), 272-281.

Sirdeshmukh, D., Singh, J. y Sabol, B. (2002). Consumer Trust, Value and Loyalty in Relational Exchanges. Journal of Marketing, 66 (1), 15-37.

Sivadas, E., y Baker-Prewitt, Jamie L., (2000). An examination of the relationship between service quality, customer satisfaction and store loyalty. Journal of Retail \& Distribution Management, 28 (2)73-82.

Sohn, C, y Tadisina, S. K. (2008). Development of e-service quality measure for internetbased financial institutions. Total Quality Management \& Business Excellence, 19, 903918.

Stiakakis, E., Georgiadis, C. K., (2009). Drivers of a tourism e-business strategy: the impact of information and communication technologies. Operational Research International Journal, 1-21

Storbacka, K., Strandvik, T. y Grönroos, C. (1994). Managing customer relationships for profit: the dynamics of relationship quality. International Journal of Service Industry Management, 5 (5), 21-38.

Straub, D., y Watson, R. (2001). Research Commentary: Transformational issues in researching IS and net-enabled organizations. Information Systems Research, 12 (4), 337-345. 
Surjadjaja, H., Ghosh, S., y Antony, J. (2003). Determining and assessing the determinants of e-service operations. Managing Service Quality, 13 (1), 39-53

Szymanski, D. M., y Hise, R. T. (2000). E-satisfaction: An initial examination. Journal of Retailing, 76 (3), 309-322.

Terblanche, N. S., y Boshoff, C. (2001). Measuring customer satisfaction with some of the controllable elements of the total retail experience: an exploratory study. South African Journal of Business Management, 32 (2), 35-41.

Vallespín, M. y Molinillo, S. (2014). El futuro de la intermediación en el sector turístico. Revista de análisis turístico, 17 (1), 13-25.

Van Riel, A. C. R., Liljander, V. y Jurriens, P. (2001). Exploring consumer evaluations of eservices: a portal site. International Journal of Service Industry Management, 12 (4), 35977.

Vázquez-Casielles, R.; Del Río-Lanza, A. y Suárez-Álvarez, L. (2009). Las agencias de viaje virtuales: ¿Cómo analizar la calidad de e-servicio y sus efectos sobre la satisfacción del cliente? Universia Business Review, Cuarto Trimestre, 122-142.

Walle, A. H. (1996). Tourism and the Internet: Opportunities for Direct Marketing. Journal of Travel Research, 35 (1), 72-77.

Wolfinbarger, M. F. y Gilly, M. C. (2001). Shopping online for freedom control and fun. California Management Review, 43 (2), 34-55.

Xiang, Z., Wöber, K. y Fesenmaier, D. R. (2008). Representation of the Online Tourism Domain in Search Engines. Journal of Travel Research, 47 (2), 137-150

Xiang, Z., Wöber, K. y Fesenmaier, D. R. (2008). Representation of the Online Tourism Domain in Search Engines. Journal of Travel Research, 47 (2), 137-150

Xiang, Z., y Gretzel, U. (2009). Role of Social Media in Online Travel Information Search. Tourism Management, 31 (2), 179-188

Yang, Z., Jun, M. y Peterson, R. T. (2004). Measuring customer perceived online service quality. Scale development and managerial implications. International Journal of Operations \& Production Management, 24 (11) 1149-1174.

Yoo, B. y Donthu, N. (2001). Developing a scale to measure the perceived quality of an Internet shopping site (SITEQUAL). Quarterly Journal of Electronic Commerce, 2 (1), 3146.

Yoon, S. J. (2002). The Antecedents and Consequences of Trust in Online Purchase Decisions. Journal of Interactive Marketing, 16 (2), 47-63.

Zeithaml, V. A., Bitner, M. J., y Gremler, D. D. (2013). Marketing de servicios, McGraw-Hill, México.

Zhilin, Y., Peterson, R.T. y Cai, S. (2003). Services quality dimensions of Internet retailing: an exploratory analysis. The Journal of Service Marketing, 17 (6/7), 685-98.

Para citar este artículo: Sarmiento Guede, J.R. (2017). La experiencia de la calidad de servicio online como antecedente de la satisfacción online: estudio empírico en los sitios web de viajes. Investigaciones Turísticas, (13), 30-53. http://dx.doi.org/10.14198/INTURI2017.13.02 